\title{
CAUSAS DE INTERNAÇÕES DE CRIANÇAS E ADOLESCENTES NOS HOSPITAIS DO SUS EM MINAS GERAIS ENTRE 1994 E 1995
}

\author{
Marcelo M. Abrantes ${ }^{1}$, Joel A. Lamounier ${ }^{2}$, Juliano F. Faria', \\ Cristiano M. Diniz', Fabiano A. F. Cunba ${ }^{1}$
}

\begin{abstract}
Resumo
Oobjetivo deste estudo foi determinar as causas mais freqüentes de internaçôes de crianças e adolescentes nos hospitais do Estado de Minas Gerais conveniados ao SUS nos anos de 1994 e 1995. As informaçôes sobre internaçôes hospitalares foram obtidas através da "Autorização de Internação Hospitalar (AIH)" que os hospitais preenchem para receberem pelos serviços. Os dados dessas AIHs são disponibilizados pelo Ministério da Savide (MS) através da rede BBS e CDROM. As variáveis estudadas foram a idade, sexo e a causa de internação. Em 1994 ocorreram 1.397.265 internaçöes e em 1995, 1.465.389. Do total de internaçôes no periodo, 18,6\% correspondem a crianças e 9,5\% a adolescentes. Predominaram as internaçôes para o sexo masculino, exceto para os individuos com idades entre os 15 e 19 anos, faixa etária em que predominaram as internaçöes relacionadas com a gestação (aproximadamente 60\%). As doencas e infecçôes do aparelho respiratório constituiram a principal causa de internação em ambos os sexos e em todas as faixas etárias, com exceção somente para o sexo feminino na faixa etária de 15 a 19 anos. A segunda causa mais freqüente correspondeu d̀s doenças gastrointestinais em todas as faixas etárias e em ambos os sexos. Conclui-se que as informaçôes do presente estudo contribuiram para o melhor conhecimento das principais causas de internaçoes em crianças e adolescentes em hospitais credenciados pelo SUS em Minas Gerais, o que permitiria a implementação de acôes e medidas preventivas do ponto de vista de saúde puiblica.
\end{abstract}

Palavras-Chave: Hospitalizaçoes; Causas de Internação; Estatísticas Hospitalares.

\begin{abstract}
Summary
The objective of this study was to determine the most frequent causes of hospital admissions of children and adolescents in the Public Health Care System in the State of Minas Gerais in 1994 and 1995. Information about hospital admissions was obtained from the Hospital Admission Authorization forms (AIHs) filled by the hospitals for reimbursement, which are available through the BBS network and a CD-ROM distributed by the Ministry of Health. The studied variables were: age, sex and cause of admission. In 1994, 1,397,265 admissions ocurred and in 1995, there were 1,465,389 admissions. During the period considered, 18,6\% of the admissions corresponded to children and 9,5\% to adolescents. Male admissions predominated except in individuals with ages between 15 and 19 years. The main causes of admissions for both sex and all ages, corresponded to diseases and infections of the respiratory system except for the 15 to 19 year interval, in which female admissions predominated, pregnacy being the most frequent admission cause (60\%). The second most frequent cause was gastrointestinal disease. It is concluded that the information of this study contributes to elucidate the main causes of admission of children and adolescents in hospital of the Public Health Care System, information that should allow the implementation of preventive actions of public health.
\end{abstract}

Key-Words: Hospital Admission; Admission Causes; Hospital Statistics.

${ }^{1}$ Bolsistas de Iniciação Cientifica CNPq.

${ }^{2}$ Professor Adjunto, Faculdade de Medicina da UFMG.

Endereço para Correspondência: Joel Alves Lamounier - Faculdade de Medicina da UFMG - Departamento de Pediatria - Av. Alfredo Balena, 190 - Belo Horizonte, MG 30130-100 / e-mail: jalamo@medicina.ufmg.br 


\section{Introdução}

As instituições de saúde representadas pelos centros de saude, hospitais e unidades básicas de sanide, caracterizam-se por oferecer assistência de caráter eminentemente curativo, com uma tendência ao uso extensivo de tecnologia de ponta e com custos cada vez mais elevados ${ }^{l}$. Dentre estas instituições o hospital é extremamente dispendioso, não só pela sua construção, mas principalmente pelo seu custeio. ${ }^{1}$

Em diferentes paises, principalmente nos subdesenvolvidos, os leitos hospitalares tornam-se necessários para garantir a recuperação da saúde, sobretudo das populações marginalizadas ou carentes. No Brasil, esta situação vem se agravando nos últimos anos, vistos o aumento desproporcionado dos custos e a falta de uma politica coerente com as necessidades e o perfil epidemiológico de nossa população. ${ }^{1}$

Existem estudos evidenciando que a assistência à saúde em escolas resultou na diminnição das taxas de internações e procura por pronto-socorros em escolares 2,3 e que programas com objetivos especificos, como a prevenção de tabagismo e gravidez, são eficazes na redução dos indices de morbi-mortalidade associados ${ }^{3,4}$. Nos Estados Unidos, um estudo mostrou que cerca de 19,0\% das internações em crianças menores de 15 anos de idade que foram, em sua maioria, devidas à pneumonia e asma, poderiam ser potencialmente evitáveis 5 .

No Brasil, informações sobre a morbimortalidade hospitalar são escassas, principalmente com relação aos hospitais conveniados com o Sistema Único de Saude (SUS). Este estudo tem o objetivo de conbecer as causas mais freqüentes de internações em crianças $e$ adolescentes nos hospitais credenciados pelo SUS de Minas Gerais. Ao se conhecerem melhor as internaçôes nesta faixa etária e suas peculiaridades, açôes preventivas e administrativas por parte dos órgãos de saúde pública do estado poderiam ser propostas para redução da incidência de doenças de causas evitáveis.

\section{Material e métodos}

Os hospitais conveniados pelo SUS, particulares, públicos e filantrópicos são instruidos para enviar regularmente as Autorizações de Internação Hospitalar (AIH) preenchidas, a fim de receberem pelos serviços prestados. Os dados contidos nestas AIHs são disponibilizados pelo Ministério da Saúde, através da rede BBS (Bulletin Board System), rede semelhante à Internet, que permite a comunicação entre computadores e troca de mensagens e dados e, mais recentemente, através de CD-ROM, que pode ser solicitado por instituiçôes de pesquisa e ensino.

Quando da realização do presente estudo, estavam disponiveis os dados até o ano de 1995, sendo então possivel e decidido analisar apenas os dois últimos anos (1994 e 1995). Nesse periodo, ocorreram 2.862.654 internações em todos os hospitais credenciados pelo SUS no Estado de Minas Gerais. Desse total, 1.397.265 internaçôes foram em 1994 e 1.465 .389 no ano de 1995. Os dados das AIHs foram preenchidos diretamente pelos hospitais e processados pelos técnicos do SUS em nivel federal. Admite-se que os procedimentos e os dados fornecidos estejam corretos. No entanto, isto não implica que fraudes contra o SUS não possam ter ocorrido com relação ao diagnóstico das internações.

Os dados, originalmente em formato DBF (Dbase III), foram convertidos para o formato REC, utilizando para este fim o software EpiInfo versão 6.02. Três variáveis foram analisadas: causas de internação (codificado pelo CID vigente), sexo e idade, nos anos base de 1994 e 1995. É importante ressaltar que as AIHs contêm outros dados, como filiação, procedência, endereço e hospital em que ocorreu o atendimento, os quais também são disponibilizados pelo MS, possibilitando análises em relação ao espaço geográfico. No entanto, essas variáveis não foram analisadas neste estudo. 
Para fins de análise foram consideradas duas categorias por faixa etária, conforme classificação adotada pela Organização Mundial da Saúde $(O M S)^{6}$ :

1 - Crianças: englobando lactentes (de 0 até 2 anos incompletos), pré-escolares (de 2 anos até 6 anos completos) e escolares (de 7 até 9 anos completos);

2 - Adolescentes: compreendendo a faixa etária de 10 a 19 anos completos. A categoria de adolescentes foi subdividida $\mathrm{em}$ duas faixas etárias: 10 a 14 anos completos e de 15 a 19 anos completos. Em todas as faixas etárias, procurou-se fazer a determinação das causas mais freqüentes de internações.

\section{Resultados}

Freqüência de internações nas diferentes faixas etárias

Na tabela 1, está distribuido o total de internações ocorridas nos anos de 1994 e 1995. Os dados estão representados em números absolutos e percentagens pelas respectivas faixas etárias. Considerando-se o período estudado, observa-se que a proporção de internações permaneceu praticamente constante nos dois anos.

No ano de 1994, 17,6\% (245.917) das internaçôes foram de crianças e 9,5\% (132.740) de adolescentes. Dados semelhantes foram observados para o ano de 1995 - 19,6\% (287.215) das internações foram de crianças $e$ 9,5\% (271.952) de adolescentes.

$O$ predominio de internaçôes foi na população de adultos e idosos, 72,9\% e 70,9\% em 1994 e 1995 respectivamente.

Com base em dados populacionais, do último censo realizado em 1991 pelo Instituto Brasileiro de Geografia e Estatística (IBGE), a população total no estado de Minas Gerais era de 15.743 .152 habitantes. ${ }^{7}$. Deste total, 5.223.096 eram crianças (0 a 9) anos e 3.420.659 adolescentes. No ano de 1994, a população de Minas Gerais aumentou para 16.327.260", podendo ser feita uma estimativa de que as internações de crianças $e$ adolescentes representaram, no ano de 1995, algo próximo de $6,5 \%$ do total da população infantil e adolescente, porcentagem esta semelhante aos dados publicados pelo Serviço de Inspeção de Saúde Nacional dos Estados Unidos, com uma taxa de 7,7\% de hospitalização para todas as faixas etárias 9 .

Freqüência de internações de crianças e adolescentes distribuídas por sexo

Na tabela 2 está ilustrada a freqüência de internações (em percentagem) de crianças $e$ adolescentes, distribuidas por sexo $e$ especificadas para cada faixa etária. O maior número de internações em crianças $e$

Tabela 1 - Total de internações por faixa etária em hospitais credenciados pelo SUS em Minas Gerais, nos anos de 1994 e 1995.

\begin{tabular}{|c|c|c|c|c|c|c|}
\hline \multirow{2}{*}{$\begin{array}{l}\text { Faixa Etária } \\
\text { (Anos) }\end{array}$} & \multicolumn{2}{|c|}{1994} & \multicolumn{2}{|c|}{1995} & \multicolumn{2}{|c|}{ Total } \\
\hline & Número & $\%$ & Número & $\%$ & Número & $\%$ \\
\hline $0-1$ & 139.726 & 10,0 & 186.104 & 12,7 & 325.830 & 11,3 \\
\hline $2-6$ & 81.041 & 5,8 & 76.200 & 5,2 & 157.241 & 5,5 \\
\hline $7-9$ & 25.150 & 1,8 & 24.911 & 1,7 & 50.061 & 1,8 \\
\hline $10-14$ & 37.726 & 2,7 & 35.170 & 2,4 & 72.896 & 2,5 \\
\hline $15-19$ & 95.014 & 6,8 & 104.042 & 7,1 & 199.056 & 7,0 \\
\hline 20 ou mais & 1.018 .606 & 72,9 & 1.038 .961 & 70,9 & 2.057 .567 & 71,9 \\
\hline Total & 1.397 .263 & 100.0 & 1.465 .388 & 100.0 & 2.862 .651 & 100.0 \\
\hline
\end{tabular}


Tabela 2 - Freqüência percentual de internações de crianças e adolescentes nas respectivas faixas etárias por sexo em hospitais conveniados com o SUS em Minas Gerais nos anos de 1994 e 1995

\begin{tabular}{ccccccc}
\hline Faixa Etária & \multicolumn{2}{c}{1994} & \multicolumn{2}{c}{1995} & \multicolumn{2}{c}{ Total } \\
Anos/Sexo & $M$ & $F$ & $M$ & $F$ & $M$ & $F$ \\
\hline $0-1$ & 55,7 & 44,3 & 50,5 & 49,5 & 52,7 & 47,3 \\
$2-6$ & 55,2 & 44,8 & 55,6 & 44,4 & 55,4 & 44,6 \\
$7-9$ & 58,0 & 42,0 & 58,3 & 41,7 & 58,1 & 41,9 \\
$10-14$ & 56,7 & 43,3 & 56,4 & 43,6 & 56,5 & 43,5 \\
$15-19$ & 21,1 & 78,9 & 18,5 & 81,5 & 19,7 & 80,3 \\
\hline
\end{tabular}

Fonte: Ministério da Sauide - rede BBS

adolescentes foi do sexo masculino, exceto na faixa etária dos 15 aos 19 anos, na qual houve predominio do sexo feminino. Nesta faixa etária, o maior número de internações do sexo feminino deveu-se à alta freqüiencia do parto $e$ problemas decorrentes da gestação.

Causas mais freqüentes de internações de crianças

Para fins de análise, o grupo de crianças foi dividido em lactentes, pré-escolares e escolares, conforme descrito na metodologia. Em cada faixa etária, determinaram-se as causas mais freqüentes de internação no periodo estudado.

Foram encontradas cerca de 750 causas de internações com freqüência menor que 2,0\%, agrupadas na categoria de "outras" para não tornar as tabelas muito extensas. $O$ objetivo, do adotar o ponto de corte com o valor mínimo de 2,0\%, foi o de estudar melhor as causas de maior frequiência e as mais comuns.

Na tabela 3 estão relacionadas as causas mais freqüentes de internaçôes nos anos de 1994

Tabela 3 - Causas mais freqüentes de internações de crianças nos anos de 1994 e 1995 em hospitais conveniados com o SUS em Minas Gerais

\begin{tabular}{|c|c|c|c|c|c|c|}
\hline \multirow[b]{2}{*}{ Causa } & \multicolumn{2}{|c|}{ Lactentes } & \multicolumn{2}{|c|}{ Pré-esco lares } & \multicolumn{2}{|c|}{ Escolares } \\
\hline & 1994 & 1995 & 1994 & 1995 & 1994 & 1995 \\
\hline $\begin{array}{l}\text { Do enças e infecçóes do aparelho } \\
\text { respirató rio }\end{array}$ & $33,4 \%$ & $27,6 \%$ & $37,9 \%$ & $37,7 \%$ & $18,3 \%$ & $17,3 \%$ \\
\hline Do enças do aparelho digestivo * & $25,2 \%$ & $20,5 \%$ & $13,6 \%$ & $13,6 \%$ & $13,4 \%$ & $13,2 \%$ \\
\hline Septicemia de tipo não especificado & $3,6 \%$ & $3,0 \%$ & $<2,0 \%$ & $<2,0 \%$ & $<2,0 \%$ & $<2,0 \%$ \\
\hline Outros recém-nascidos de pré-termo & $3,4 \%$ & $2,6 \%$ & $<2,0 \%$ & $<2,0 \%$ & $<2,0 \%$ & $<2,0 \%$ \\
\hline $\begin{array}{l}\text { Hérnia inguinal sem menção de } \\
\text { obstrução o u gangrena }\end{array}$ & $<2,0 \%$ & $<2,0 \%$ & $2,4 \%$ & $2,5 \%$ & $2,4 \%$ & $2,2 \%$ \\
\hline $\begin{array}{l}\text { Glo merulo ne frite aguda não } \\
\text { especificada }\end{array}$ & $<2,0 \%$ & $<2,0 \%$ & $<2,0 \%$ & $<2,0 \%$ & $2,1 \%$ & $<2,0 \%$ \\
\hline $\begin{array}{l}\text { Outras (causas com incidência menor } \\
\text { que } 2,0 \% \text { ) }\end{array}$ & $34,4 \%$ & $46,3 \%$ & $46,1 \%$ & $46,2 \%$ & $63,8 \%$ & $65,3 \%$ \\
\hline
\end{tabular}


e 1995 para lactentes, pré-escolares e escolares, dispostos em valores percentuais. Procurouse fazer uma avaliação da tendência das internações, isto é, se bouve aumento ou decréscimo no total, nos dois anos estudados. $O$ somatório das causas principais de internações, com freqüência acima de 2,0\% foi de 70,0\% no ano de 1994 e 50,0\% no ano de 1995.

Para facilitar a análise por grupos, a broncopneumonia, a pneumonia e a asma foram agrupadas como "doenças $e$ infecções do aparelho respiratório". O mesmo ocorreu com as doenças do aparelho digestivo e neste item as causas variaram entre as diversas faixas etárias. A causa de internação classificada pelo CID como "depleção de volume líquido" foi considerada como uma conseqüência de doenças do aparelho digestivo.

Causas mais freqüientes de internações de adolescentes

Conforme descrito anteriormente na metodologia, o grupo de adolescentes foi dividido, em duas faixas etárias: de 10 a 14 anos $e$ de 15 a 19 anos. Para cada subgrupo etário determinaram-se as causas mais freqüentes de internações no periodo estudado, utilizando-se como critério uma freqüência minima de 2,0\% para serem incluidas na tabela.
Na tabela 4, estão os dados referentes às internações no período de 1994 e 1995 de adolescentes do sexo masculino no subgrupo de 10 a 14 anos e subgrupo de 15 a 19 anos respectivamente. Como já mencionado antes, algumas causas, com freqüência inferior a 2,0\% não apareceram no ano seguinte. $O$ somatório das causas principais de internações, ou seja, aquelas com incidência acima deste percentual, variou entre $18,0 \%$ e 27,0\%. Assim, as internações, em sua maioria (73,0 a 82,0\%) foram devidas a diferentes causas e com baixa freqüência $(<2,0 \%)$, dificultando a apresentação dos dados específicos nas tabelas.

Constatamos que a apendicite aguda, sem menção de peritonite, surge como uma causa cirúrgica com freqüência maior que 2,0\% em adolescentes.

$\mathrm{Na}$ tabela 5, observamos as causas mais freqüentes de internações de adolescentes do sexo feminino no periodo de 1994 a 1995. Observa-se que as infecçóes do aparelho respiratório (broncopneumonia e pneumonia) representaram o maior indice de internações na faixa etária de 10-14 anos e que o parto representa uma importante causa de internação em adolescentes, principalmente entre 15 e 19 anos de idade.

Tabela 4 - Causas mais freqüentes de internações de adolescentes do sexo masculino nos anos de 1994 e 1995 em hospitais conveniados com o SUS em Minas Gerais

\begin{tabular}{l|cr|rr}
\hline \multicolumn{1}{c|}{ Causa } & \multicolumn{2}{c|}{10 a 14 anos } & \multicolumn{2}{c}{15 a 19 anos } \\
& 1994 & 1995 & 1994 & 1995 \\
\hline Doenças e infecçóes do aparelho respiratório & $14,2 \%$ & $2,2 \%$ & $10,5 \%$ & $9,6 \%$ \\
Doenças do aparelho digestivo * & $7,9 \%$ & $7,7 \%$ & $2,3 \%$ & $<2,0 \%$ \\
Apendicite aguda sem menção de peritonite & $2,1 \%$ & $2,5 \%$ & $<2,0 \%$ & $2,4 \%$ \\
Hérnia inguinal sem menção de obstrução ou de & $2,1 \%$ & $2,0 \%$ & $2,4 \%$ & $2,5 \%$ \\
gangrena & & & & \\
Outras (causas com incidência menor que 2,0\%) & $73,7 \%$ & $85,6 \%$ & $82,8 \%$ & $83,5 \%$ \\
\hline * Colite, enterite, gastroenterite, deplecão de rolume líqido
\end{tabular}

* Co lite, enterite, gastro enterite, depleção de volume líquido

Fonte: Ministério da Saúde - rede BBS 
Tabela 5 - Causas mais freqüentes de internações de adolescentes do sexo feminino nos anos de 1994 e 1995 em hospitais conveniados com o SUS em Minas Gerais

\begin{tabular}{lrrrr}
\hline \multicolumn{1}{c}{ Causa } & \multicolumn{2}{c}{10 a 14 anos } & \multicolumn{2}{c}{15 a 19 anos } \\
& 1994 & 1995 & 1994 & 1995 \\
\hline Doenças e infecçoes do aparelho respiratório & $12,3 \%$ & $11,6 \%$ & $<2,0 \%$ & $<2,0 \%$ \\
Parto & $7,0 \%$ & $5,8 \%$ & $44,9 \%$ & $42,4 \%$ \\
Doenças do aparelho digestivo * & $3,2 \%$ & $4,1 \%$ & $<2,0 \%$ & $<2,0 \%$ \\
Parto cesáreo & $<2,0 \%$ & $<2,0 \%$ & $7,1 \%$ & $6,2 \%$ \\
Aborto & $<2,0 \%$ & $<2,0 \%$ & $2,6 \%$ & $2,4 \%$ \\
Outras (causas com incidência menor que 2,0\%) & $77,5 \%$ & $77,2 \%$ & $45,4 \%$ & $49,0 \%$ \\
\hline
\end{tabular}

* Colite, enterite, gastro enterite, diarréia infecciosa e depleção de volume líquido

Fonte: Ministério da Saúde - rede BBS

Distribuição sazonal das causas mais freqüentes de internações

A causa mais freqüente de internação em cada mês do periodo estudado foi determinada e analisada para verificar uma possível distribuição sazonal.

Nas crianças, as principais causas de internações foram as doenças infecciosas do aparelho respiratório e, dentre elas, a mais freqüente foi a broncopneumonia por microorganismo não especificado. $A$ distribuição mensal de internaçôes, motivadas por esta causa, englobando ambos os sexos, variou de $10,6 \%$ a $11,2 \%$ nos dois anos de estudo. Observou-se uma tendência de aumento de internações nos meses de inverno (maio a agosto), com picos de 18,6\% e 21,0\%, assim como um aumento global no ano de 1995.
No grupo dos adolescentes, considerando ambos os sexos $e$ as duas faixas etárias, o parto constituiu o motivo principal de internação. Na figura 1 observamos a incidência mensal que variou entre $20,5 \%$ a $27,3 \%$ nestes dois anos, com uma tendência de aumento a partir do segundo semestre de 1995, apresentando um pico máximo de $28,6 \%$ entre os meses de agosto a outubro.

Distribuição das causas de internação por faixas etárias

As causas de internação na infância são menos diversificadas do que na adolescência, a maioria por infecçôes do aparelho respiratório e doenças do aparelho digestivo, que diminuem de freqüência progressivamente com a idade (Figura 2).

Figura 1 - Distribuição sazonal de freqüência de internações "por parto" de adolescentes nos anos de 1994 e 1995 nos hospitais credenciados com o

\section{SUS em Minas Gerais}

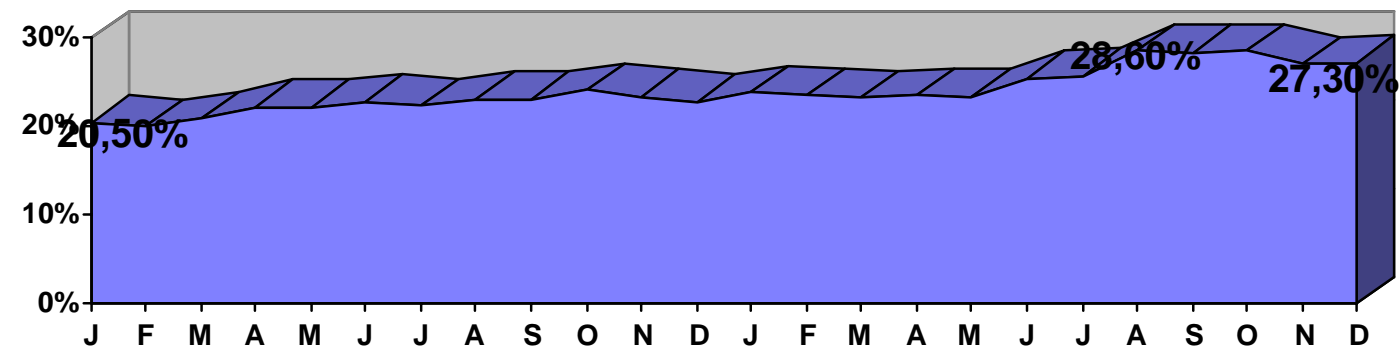

Fonte: Ministério da Saúde - rede BBS 
Figura 2 - Perfil da nosologia nas diversas faixas etárias em hospitais conveniados com o SUS em Minas Gerais nos anos de 1994 e 1995

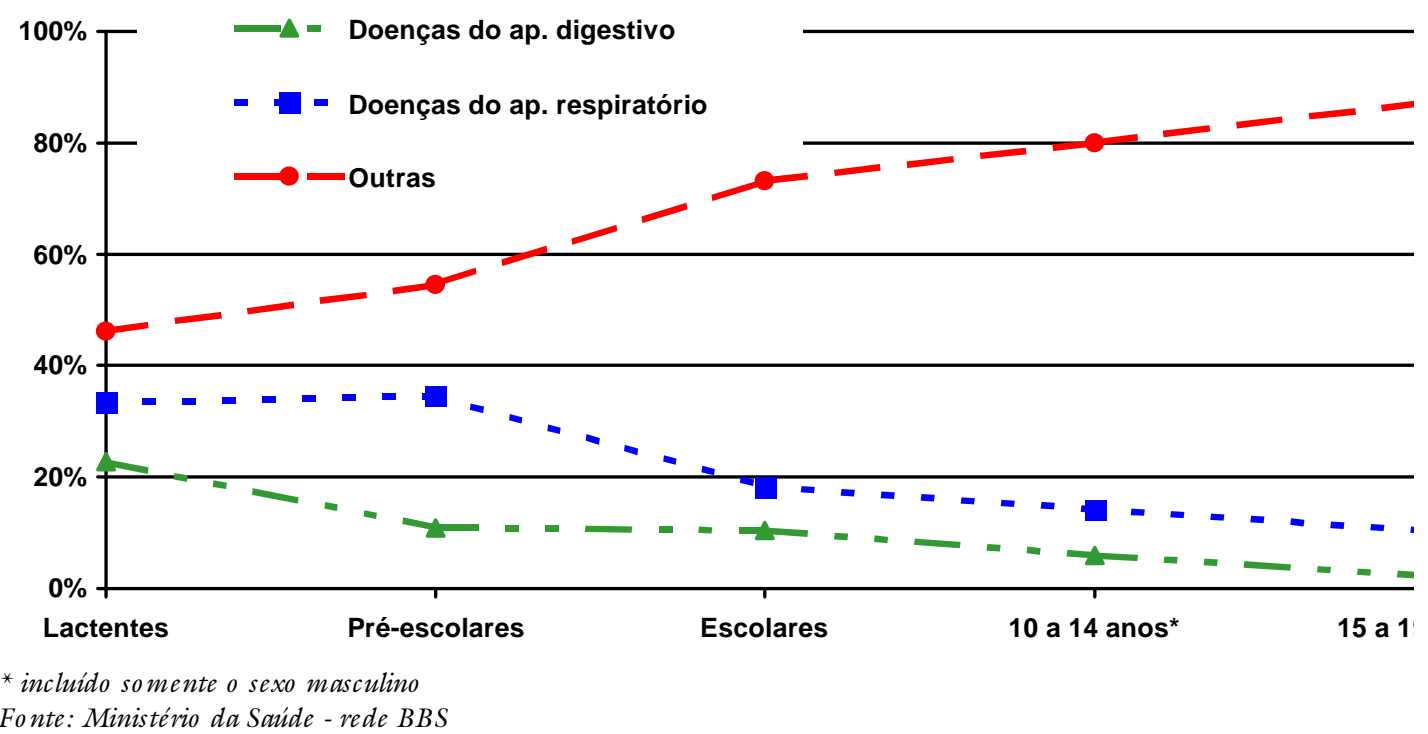

Nas faixas etárias de 10-14 e 15-19 anos, os dados são referentes ao sexo masculino, uma vez que o parto e problemas relacionados com a gestação são especificos do sexo feminino $e$ pela sua alta freqüência poderiam interferir na análise das causas de internações do sexo masculino.

\section{Discussão e conclusões}

Este estudo mostrou que as internaçôes se apresentaram com maior freqüência nas crianças $(18,6 \%)$ do que nos adolescentes (9,5\%), sendo os lactentes a faixa etária mais acometida (11,3\%). Dados semelhantes foram encontrados na população da região de Ribeirão Preto, com as crianças sendo responsáveis por $18,7 \%$ e os adolescentes por $10,4 \%^{1}$ das internações.

Em todas as faixas etárias, o maior número de internaçôes, tanto no ano de 1994 como no de 1995, foram do sexo masculino, exceto na faixa de 15 a 19 anos. A maior prevalência do sexo masculino tem sido descrita também em outros estudos ${ }^{1,10}$. Entre as possíveis explicações para um maior predominio de internaçôes em meninos até a idade de 14 anos, estaria a maior exposição a agentes infecciosos e a traumas, pois há maior liberdade de ações e brincadeiras no sexo masculino em comparação com o sexo feminino por razóes sócioculturais em nosso meio.

Por outro lado, a maior freqüência de internações no sexo feminino após os 15 anos está diretamente ligada à gravidez na adolescência. Predominio este confirmado por outros estudos que também relacionaram maior incidência relacionada à gravidez ${ }^{1,10}$, confirmando portanto, o que os outros estudos têm mostrado, ou seja, um aumento crescente no número de mães adolescentes em diferentes paises e em todas as camadas sociais. $\mathrm{Na}$ América Latina, a gestação na adolescência está relacionada com altas taxas de morbimortalidade perinatal e infantil em decorrência de vários fatores ${ }^{11}$. Estatísticas de registro civil do IBGE de 1990 mostraram que, de um total de 2.419 .927 registros, 8.340 (0,3\%) eram partos de mães adolescentes com idade inferior aos 15 anos e 379.873 (15,7\%) eram partos de adolescentes com idades entre os 15 e os 19 anos $^{12}$. Num estudo na região de Oxford, Inglaterra, $33,3 \%$ das gestações eram em 
mulheres com menos de 20 anos $^{10}$. No Brasil, no estudo de Nóbrega e colaboradores, o indice de gestações em adolescentes foi de $14,5 \%{ }^{13}$. $O$ número de partos vem aumentando entre as adolescentes, conforme mostram também outros estudos $^{14,15,16}$. Na Santa Casa de Belém, de 1.000 nascimentos, $28,0 \%$ correspondem a mães adolescentes, e 2,8\% tinham idade inferior a 16 anos $^{17}$. No Hospital das Clinicas da Universidade Federal de Minas Gerais, de um total de 912 partos, 112 foram de adolescentes, representando $12,2 \%{ }^{18}$. Em estudo realizado na Maternidade Odete Valadares, da rede pública de Belo Horizonte, de um total de 5.989 partos ocorridos no ano de 1992, 20,8\% foram de adolescentes $^{19}$.

Considerando que, entre os 15 e os 19 anos, $80,0 \%$ das internações são do sexo feminino e que 60,0\% são por problemas relacionados à gestação, concluimos que essas causas chegam a ser responsáveis por até 50,0\% do total das internaçôes de adolescentes entre 15 e 19 anos.

Baseados na alta incidência de gestação em adolescentes, na desinformação em relação às questões da sexualidade e altos indices de iniciação sexual precoce $e^{20}$ e na evidência de que medidas de orientação sexual são efetivas na redução de indices de gestação $\mathrm{em}$ adolescentes ${ }^{3}$, reafirma-se a necessidade de orientação sexual de rotina nas consultas médicas de adolescentes e implantação dessa orientação no currículo escolar.

Com relação às doenças entre as crianças, todas as causas de internações declinaram com o aumento da idade exceto a asma, que teve seu pico na faixa etária entre $2 e$ os 7 anos de idade. As infecçôes do aparelho respiratório (broncopneumonia, pneumonia, etc.) foram a principal causa de internação, apresentando um caráter sazonal evidente, com maior incidência nos meses de inverno. Dados semelhantes também são relatados em outros estudos $^{21}$. Em uma clínica particular de São Paulo, em um estudo envolvendo 630 adolescentes pertencentes às classes sociais alta ou média (330 do sexo masculino e 300 do sexo feminino), as doenças do aparelho respiratório foram um dos motivos principais de consulta médica ${ }^{22}$. Embora estas populações sejam de estratos sócioeconômicos diferentes, podemos notar que as doenças do aparelho respiratório representam o grupo mais prevalente de morbidade. Admite-se que as doenças respiratórias podem estar acometendo de forma semelhante populaçôes diferentes em virtude de fatores climáticos.

As doenças respiratórias são importantes e, em geral aumentam de incidência em decorrência dos fatores climáticos favorecidos pela piora da qualidade do ar e de condições de moradia em grandes centros urbanos.

$A$ partir do conbecimento de que essas causas são responsáveis por grande parte das internações, principalmente em crianças, podemos realizar um atendimento em nivel primário mais eficaz visando a prevenção de suas complicações. Estudos já comprovaram que $o$ atendimento primário é um fator de melhor prognóstico, evitando-se as internações hospitalares ${ }^{5}$.

O segundo grupo de causas mais freqüentes de internações em crianças $e$ adolescentes do sexo masculino, as doenças do aparelho digestivo, tem sido relatado também em outros estudos. Em Israel, em uma unidade pediátrica de observação e tratamento, as causas digestivas foram responsáveis por $24,0 \%$ dos procedimentos $^{21}$.

Pode-se concluir que as informações do presente estudo contribuiram para melhor conbecimento das principais causas de internações em crianças e adolescentes em hospitais credenciados pelo SUS em Minas Gerais. Do ponto de vista de saúde pública, açôes $e$ medidas preventivas como vacinação, intensificação do atendimento primário, tratamento ambulatorial, orientação populacional e educação sexual precisam ser reforçadas no sentido de reduzir os indices de 
morbi-mortalidade na infância e adolescência. Um bom exemplo foi a recente epidemia de sarampo, que poderia ser prevenida com a vacinação, que tem resultado em elevada morbidade e com repercussóes nas taxas de ocupação de leitos hospitalares do SUS. Os recursos econômicos gastos com internações hospitalares poderiam ser revertidos e aplicados nessas ações básicas de saúde, reconbecidamente eficazes como agentes importantes na melhoria dos indicadores de saúde da população.

\section{Agradecimentos}

Ao Dr. Fulgêncio José Gazzinelli Abrantes e ao SUS/MG pelas informações $e$ dados da rede BBS. Ao CNPq e FAPE-MIG pelo apoio financeiro.

\section{Bibliografia}

1.D’Oleo RJM, Fávero M. Perfil sociodemográfico da população que demanda assistência médico hospitalar em região do Estado de São Paulo, Brasil, 1988. Revista de Saúde Pública 26:256-63, 1992.

2. Santelli J, Kouzis A, Newcomer S. Schoolbased bealth centers and adolescent use of primary care and hospital care. Journal of Adolescent Health 19:267-275, 1996.

3. Zabin LS. Evaluation of a pregnancy prevention program for urban teenagers. Fam Plann Perspect 18:119-26, 1986.

4. Kottke TE, Battista RN, DeFriese GH, et al. Attributes of sucessful smoking cessation interventions in medical practice: a metaanalysis of 39 controlled trials. Journal of American Medical Association 259:2883-89, 1988.

5. Pappas G, Hadden WC, Kozak LJ, et al. Potentially Avoidable Hospitalizations: Inequalities in Rates between US Socioeconomic Groups. American Journal of Public Health 87:811-16, 1997.

6. Organización Mundial de la Salud. El embarazo y el aborto en la adolescencia. Serie de Informes Tecnicos, $N^{\circ}$ 583, 1975.
7. MINAS GERAIS, Anuário Estatístico. 1:146, 1995.

8. BRASIL, Instituto Brasileiro de Geografia e Estatística. Dados não publicados.

9. EUA, National Center for Health Statistics. Current estimates of the National Health Interview Survey, 1992. Vital and Health Statistics, Series 10, 189:119, 1994.

10.Henderson JH, Goldacre M, Yeates D. Use of hospital inpatient care in adolescence. Archives of Disease in Childhood 69:559-563, 1993.

11.Organización Mundial de la Salud. El embarazo y el aborto en la adolescencia. Série de Informes Técnicos, $N^{0}$ 5834, 1988.

12.BRASIL, Instituto Brasileiro de Geografia e Estatística. Estatísticas do registro civil. 17, 1990.

13. Nóbrega FJ. Antropometria, patologias e malformaçôes congênitas do recém-nascido brasileiro e estudos de associação com algumas variáveis maternas. Jornal de Pediatria 59:144, 1985.

14.Espiridião, S. Avaliação dos recém-nascidos de mães adolescentes no H.M. de Santo André. Pediatria Moderna 28:526-529, 1992.

15.Bhering CA, Bhering JA, Mendonça EG. Estudo descritivo e comparativo de adolescentes grávidas e seus conceptos no municipio de Barra Mansa, RJ. Arquivo Brasileiro de Pediatria 1:47-52, 1994.

16.Sá DSS, Silva MFPR, Magalhães MAS, Epifanio Netto A, Rego TMS. O perfil da mãe adolescente e do seu filho no hospital maternidade Carmela Dutra, Rio de Janeiro. Arquivo Brasileiro de Pediatria 3: 139 142, 1996.

17. Costa MCO, Bittar K, Bernardino C. Antropometria do recém-nascido $e$ associação com variáveis maternas. Faculdade Estadual de Medicina do Pará, Belém, PA, 1992. 
18.Rego MAS, Anchieta LM \& Lamounier JA Caracteristicas de gestantes adolescentes atendidas no Hospital das Clinicas da UFMG. In: Anais XXVII Congresso Brasileiro de Pediatria p.58, 1991.

19. Menezes IM, Pereira MVC, Resende MA, Lamaita RM, Santos JN, Silveira SM, Freitas AHA, Cardoso AM, Lamounier JA. Avaliação das condições perinatais em Maternidade pública de Belo Horizonte. Revista Médica de Minas Gerais 3; $65,1993$.
20. Costa MCO, Pinho JFC, Martins SJ. Aspectos psicossociais e sexuais de gestantes adolescentes em Belém, Pará. Jornal de Pediatria 71:151-157, 1995.

21. Variations in rates of hospitalization of children (letter; comment). New England Journal of Medicine 322: 206-207, 1990.

22.Crespin J. Adolescência. Reflexões sobre o motivo da consulta de 630 jovens. Nestlé Serviço de Informação Cientifica. Temas de Pediatria 35:19, 1989. 